\title{
LETTERS
}

\section{Evidence-Based Precision Medicine is Needed to Move Toward General Internal Precision Medicine}

\author{
Evrim Jaccard, $M D, P h D^{1,2}$, Jacques Cornuz, $M D^{2}$, Gérard Waeber, $M D^{7}$, and ldris Guessous, \\ $M D, P h D^{2,3}$
}

'Department of Internal Medicine, BH10-628, Internal Medicine, Lausanne University Hospital (CHUV), Lausanne, Switzerland; ${ }^{2}$ Department for Ambulatory Care and Community Medicine, University of Lausanne, Lausanne, Switzerland; ${ }^{3}$ Unit of Population Epidemiology, Division of Primary Care Medicine, Department of Community Medicine, Primary Care and Emergency Medicine, Geneva University Hospitals, Geneva, Switzerland.

J Gen Intern Med 33(1):11-2

DOI: $10.1007 / \mathrm{s} 11606-017-4149-0$

(c) Society of General Internal Medicine 2017

$\mathrm{T}$ he terms individualized, predictive, personalized, and precision have all been used to characterize a new paradigm in medicine (hereinafter referred to as precision medicine). ${ }^{1}$ However, these terms have different meanings, and their use may impact how the medical community views this new field of medicine. ${ }^{2}$ This may be particularly important in the area of general internal medicine, given the skepticism of many general practitioners (GPs), ${ }^{3-6}$ likely fueled by the paucity of original research in an ocean of editorials and commentaries. Recent trend analysis of terms and research types in general internal precision medicine is currently lacking.

To this end, therefore, we assessed the evolution of the terminology used and the amount of original research published overall and in ten major general internal medicine (GIM) journals.

We searched PubMed using the four specific terms mainly used to characterize precision medicine, namely individualized, predictive, personalized, and precision. Both free text and medical subject headings (i.e. MeSH terms) were used, and the search was limited to the period between January 2000 (3 years before the completion of the Human Genome Project) and December 2016, with no other filters.

During this period, the terms predictive and individualized medicine were identified in 179 and 495 articles, respectively, while 6775 and 10,830 articles were retrieved for personalized and precision medicine, respectively. Until 2009, the most frequently used term in the literature was personalized medicine; since 2009, precision medicine has been the most common (Fig. 1a). When the search was limited to publications in the ten major GIM journals, we found that precision medicine was the most widely used term (Fig. 1b). After reviewing the titles and abstracts of the 189 articles retrieved in the ten major GIM journals, only 153 were considered to really encompass aspects related to precision medicine, among which just eight were original articles (Fig. 1c). The number of original articles per journal, along with the publication year and the term used in the original research publication, are indicated in Figure 1d.

While the number of publications in precision medicine has increased since the completion of the Human Genome Project, a clear change in the terminology used can be observed beginning in 2008/2009, in which the term precision replaced the terms personalized and individualized. Of note, the term personalized has generally not been used in articles published in the ten major GIM journals. One reason might be the widespread opinion among GPs that medical care is by nature individualized or personalized to the needs of the patients. Our work also shows that despite a dramatic increase in the number of publications, the number of original research articles published in the ten selected GIM journals remained consistently and dramatically low. The disproportionate ratio of non-original (editorial, commentary, etc.) to original research articles might contribute to the skepticism on the part of GPs. We strongly believe that GPs have a key role in translating precision medicine for patients, especially in informing them about precision medicine, implementing appropriate precision care and avoiding inappropriate care, and sharing risk/benefit information. GPs should engage in moving this new field from "the right drug for the right patient" to "the right precision medicine for all our patients." At this time, however, most work in this area is speculative. Additional evidence-based precision medicine that can be translated to clinical practice will be necessary to move GPs toward embracing the concept of general internal precision medicine. 
a

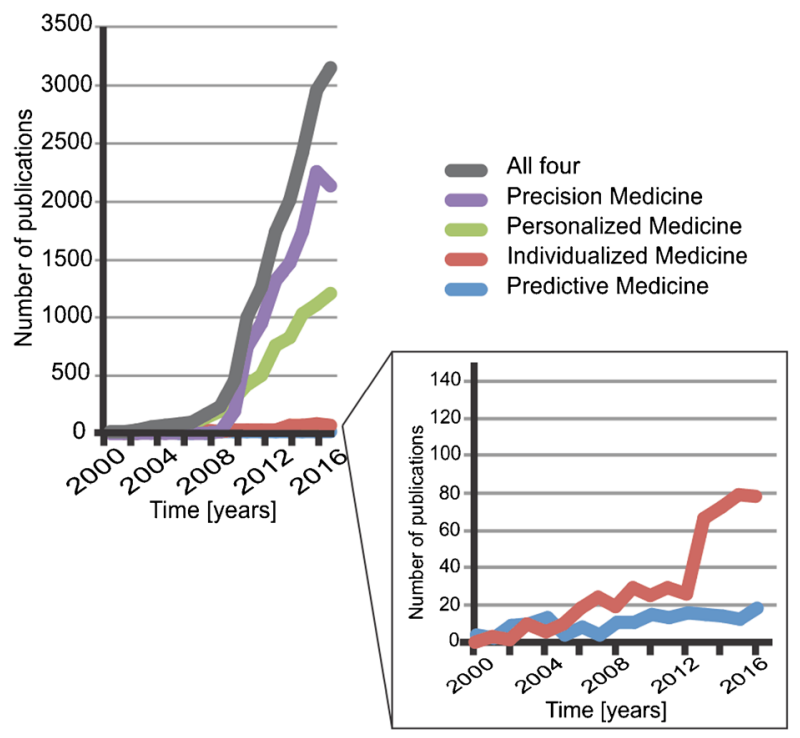

b

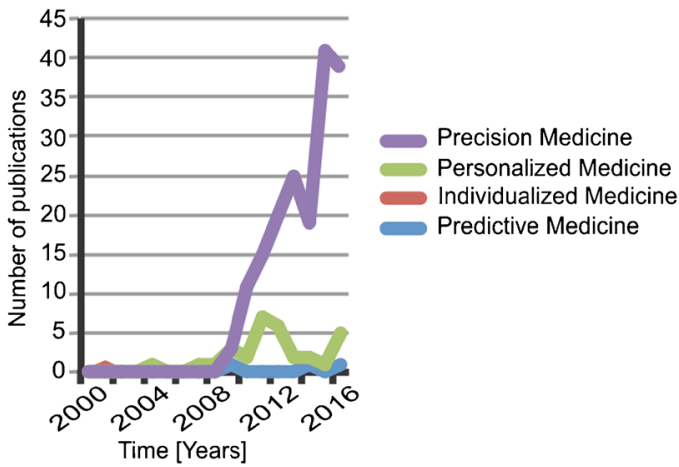

C

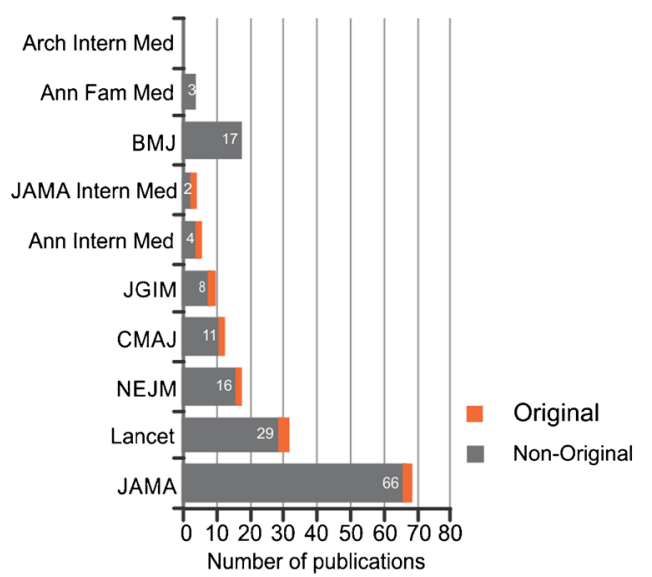

d

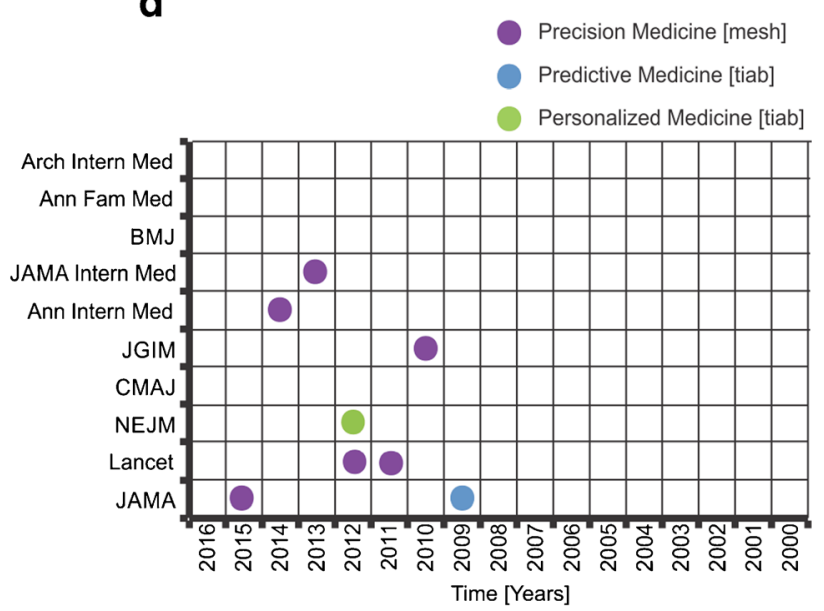

Figure 1 Landscape of publications on precision medicine overall and in ten major GIM journals. a Evolution of terminology in precision medicine. b Evolution of terminology in the ten main GIM journals. $\mathbf{c}$ Number of original articles on precision medicine published by ten major GIM journals. $d$ Number and year of original publications in ten major GIM journals.

Corresponding Author: Idris Guessous, MD, PhD; Department for Ambulatory Care and Community MedicineUniversity of Lausanne, Lausanne, Switzerland (e-mail: Idris.guessous@chw.ch).

Contributors: All contributors to this work are listed in the author byline.

Funding: This work was funded in part by the Health 2030 initiative.

\section{Compliance with Ethical Standards:}

Prior Presentations: This work has not been presented previously.

Conflict of Interest: Idris Guessous is deputy editor at JGIM. The other authors declare that they have no conflict of interest.

\section{REFERENCES}

1. Ashley EA. The precision medicine initiative: a new national effort. JAMA 2015;313(21):2119-2120. doi:10.1001/jama.2015.3595.

2. Khoury, MJ. The Shift From Personalized Medicine to Precision Medicine and Precision Public Health: Words Matter! https://blogs.cdc.gov/genomics/2016/04/21/shift/. Genomics Health Impact Blog. https://blogs. cdc.gov/genomics/2016/04/21/shift/. Accessed June 30, 2017.

3. Joyner MJ, Paneth N. Seven Questions for Personalized Medicine. JAMA 2015;314(10):999-1000. doi:10.1001/jama.2015.7725.

4. Khoury MJ, Galea S. Will Precision Medicine Improve Population Health? JAMA 2016;316(13):1357-1358. doi:10.1001/jama.2016.12260.

5. Hunter DJ. Uncertainty in the Era of Precision Medicine. N Engl J Med 2016;375(8):711-713. doi:10.1056/NEJMp1608282.

6. Jameson JL, Longo DL. Precision medicine-personalized, problematic, and promising. N Engl J Med 2015;372(23):2229-2234. doi:10.1056/ NEJMsb1503104. 